\title{
Qualitative link between work of adhesion and thermal conductance of metal/diamond interfaces
}

\section{Citation}

Monachon, Christian, Georg Schusteritsch, Efthimios Kaxiras, and Ludger Weber. 2014. "Qualitative Link between Work of Adhesion and Thermal Conductance of Metal/Diamond Interfaces." Journal of Applied Physics 115 (12): 123509. https://doi.org/10.1063/1.4869668.

\section{Permanent link}

http://nrs.harvard.edu/urn-3:HUL.InstRepos:41384033

\section{Terms of Use}

This article was downloaded from Harvard University's DASH repository, and is made available under the terms and conditions applicable to Other Posted Material, as set forth at http:// nrs.harvard.edu/urn-3:HUL.InstRepos:dash.current.terms-of-use\#LAA

\section{Share Your Story}

The Harvard community has made this article openly available.

Please share how this access benefits you. Submit a story.

Accessibility 


\title{
Qualitative link between work of adhesion and thermal conductance of metal/diamond interfaces
}

\author{
Christian Monachon, ${ }^{1, a)}$ Georg Schusteritsch, ${ }^{2, b)}$ Efthimios Kaxiras, ${ }^{3}$ and Ludger Weber ${ }^{1}$ \\ ${ }^{1}$ Laboratoire de Métallurgie Mécanique, Ecole Polytechnique Fédérale de Lausanne, Lausanne, Switzerland \\ ${ }^{2}$ School of Engineering and Applied Sciences, Harvard University, Cambridge, Massachusetts 02138, USA \\ ${ }^{3}$ Department of Physics and School of Engineering and Applied Sciences, Harvard University, Cambridge, \\ Massachusetts 02138, USA
}

(Received 20 November 2013; accepted 16 March 2014; published online 26 March 2014)

\begin{abstract}
We report Time-Domain ThermoReflectance experiments measuring the Thermal Boundary Conductance (TBC) of interfaces between diamond and metal surfaces, based on samples consisting of [111]-oriented diamond substrates with hydrogen or with $\mathrm{sp}^{2}$ carbon surface terminations created using plasma treatments. In a concurrent theoretical study, we calculate the work of adhesion between $\mathrm{Ni}, \mathrm{Cu}$, and diamond interfaces with (111) surface orientation, with or without hydrogen termination of the diamond surface, using first-principles electronic structure calculations based on density functional theory (DFT). We find a positive correlation between the calculated work of adhesion and the measured conductance of these interfaces, suggesting that DFT could be used as a screening tool to identify metal/dielectric systems with high TBC. We also explain the negative effect of hydrogen on the thermal conductance of metal/diamond interfaces. (C) 2014 AIP Publishing LLC. [http://dx.doi.org/10.1063/1.4869668]
\end{abstract}

\section{INTRODUCTION}

Interfaces can have a substantial impact on heat conduction in systems as varied as composites for thermal management, ${ }^{1,2}$ superlattices, ${ }^{3,4}$ or microelectronic devices. ${ }^{5-8}$ They also allow for exceptionally low crossplane conductances in bulk layered materials. ${ }^{9,10}$ The fact that the conductance at an interface, referred to as Thermal Boundary Conductance (TBC), is finite, is responsible for the whole range of behavior observed at interfaces. The TBC is mainly governed by properties of the materials on either side of the interface, but several features of the interface itself can also have a substantial influence on the resulting performance. Examples of such features include: intermixing between the layer and substrate, ${ }^{11}$ ion bombardment of the interface, ${ }^{12,13}$ roughness, ${ }^{14,15}$ and chemical termination of the substrate, ${ }^{16-19}$ all of which have an impact on the TBC. Recently, Losego et al. showed that there is a link between the chemical bonding at an interface between gold and quartz and the TBC of this interface by adding Self Assembled Monolayers (SAMs) between these two materials. ${ }^{20}$ They used Time-Domain ThermoReflectance (TDTR) measurements to determine the TBC of interfaces with various SAM compositions and link these results with a laser spallation method to determine the strength of bonding at the interface. They found that the stronger the adhesion of gold films on a quartz substrate, the higher the TBC is. This suggests that adhesion represents another way of influencing the TBC.

\footnotetext{
a) Author to whom correspondence should be addressed. Email address: christian.monachon@gmail.com

${ }^{b}$ Present address: Department of Physics and Astronomy, University College London, Gower Street, London WC1E 6BT, United Kingdom.
}

Adhesion of thin films on substrates can be measured experimentally in various ways, e.g., by direct pull-off techniques, ${ }^{21-23}$ blister tests, ${ }^{22,24}$ laser spallation, ${ }^{20}$ film buckling $22,25,26$ or scratch tests. ${ }^{27}$ Experimental details, such as finding a way to glue the pull-out pad more strongly than the interface, reduce the range of adhesion energy values that can be obtained by the first methods. On the other hand, the scratch test yields excellent qualitative results that are hard to link directly to the actual adhesion energy as plastification of the film impacts the energy release of the film. ${ }^{28}$ Moreover, none of these techniques make sure that the delamination occurs at the interface and not in the few layers surrounding it. ${ }^{29}$ An altogether different approach that does not suffer from these issues is to employ theoretical methods such as calculations within the framework of DensityFunctional-Theory (DFT) to address the problem of adhesion. $^{30,31}$

The metal/diamond interface has already been shown to exhibit a wide variety of TBC depending on the diamond surface termination ${ }^{17,19}$ and orientation. ${ }^{32}$ A similar trend has been reported on the $\mathrm{Al} /$ graphene interface. ${ }^{33} \mathrm{H}$ has been shown to diminish the adhesion force between diamond and aluminum. ${ }^{30}$ In this work, we explore the link between the work of adhesion $\left(W_{\mathrm{adh}}\right)$ between $\mathrm{Cu}$ or $\mathrm{Ni}$ thin films and a diamond substrate calculated using DFT and the TBC of these interfaces as measured by TDTR. We also extend this analysis to the $\mathrm{Al}$ and $\mathrm{Ti} /$ diamond systems since the values of $W_{\text {adh }}$ in these cases already exist in the literature. ${ }^{30,31}$ Even though the interfacial stiffness is the quantity expected to influence directly the passage of phonons through a metal/diamond interface, the work of adhesion is the quantity preferred in this study as it is easier to measure. Moreover, as long as plasticity is not considered, the work of adhesion should roughly scale with the bonding stiffness. 


\section{METHODS}

\section{A. Experiment}

\section{Experimental setup}

The experimental setup used in this paper is a coaxial two tints pump/probe experiment ${ }^{34}$ and has been described in detail previously. ${ }^{19,35}$ This setup uses a Spectra Physics Tsunami femtosecond laser producing pulses of about $200 \mathrm{fs}$ and $785 \mathrm{~nm}$ at a $80 \mathrm{MHz}$ repetition rate. Its beam is split into two parts, one used to heat up the sample surface (the pump), and one to test the reflectivity of the sample surface (the probe). The pump beam passes via a mechanical delay stage and the probe beam follows a path of about the same yet fixed length. Both beams are color-filtered using a sharp longpass (pump, $790 \mathrm{~nm}$ ) and shortpass (probe, $780 \mathrm{~nm}$ ) filter. Both beams are then focused on the same spot of about $5 \mu \mathrm{m} e^{-2}$ radius on the sample's surface. Varying the length of the optical path of the pump part allows for the creation of a delay between the arrival of the pump and probe on the sample surface from 0 to $4.02 \mathrm{~ns}$. The pump beam is also modulated with an electro-optic modulator at a frequency of 10.7 $\mathrm{MHz}$ to enable lock-in amplification of the resulting signal. After passing through the same shortpass filter as previously described to decrease the influence of stray light from the pump beam, the probe signal is monitored using a fast photodiode, then passed through a frequency filter centered around $10.7 \mathrm{MHz}$ and fed into a pre-amplifier before lock-in detection.

The $\mathrm{X} / \mathrm{Y}$ ratio of the values measured by the lock-in was recorded rather than just the $\mathrm{X}$-signal, because it substantially decreases the impact of a change in the overlap of the pump and probe spots. ${ }^{36}$ The size of both spots was measured using a CMOS camera (placed in the beam line using a flipping mirror) at 0 and $4 \mathrm{~ns}$ in order to take into account a change in spot size over the course of the delay stage. We used fluences of about $0.6 \mathrm{~mJ} \mathrm{~cm}{ }^{-2}$, which means that the sample surface was heated up by a few $\mathrm{K}$ at most. A background signal was recorded after each measurement to extract any influence of stray light coming from the pump beam or of electronic noise.

\section{TBC measurement}

For the measurement of TBC, datasets containing X/Y ratio over delay times from 0 to $4.02 \mathrm{~ns}$ were recorded. The obtained experimental data were fitted using the model first proposed by Cahill ${ }^{36,37}$ with TBC between metal and diamond substrate as a free parameter. ${ }^{19,38}$ The thermal model used did not include the TBC between the $\mathrm{Al}$ suscepting layer and the metal layer since the TBC values of metalmetal interfaces is typically one order of magnitude higher ${ }^{12}$ than that of metal-dielectric ones; and therefore, it should not have a substantial influence on the measurement.

Diamonds were purchased from Element 6 (Shannon Airport, Shannon, Co. Clare, Ireland, reference MD111/11). The stones were mounted on a sample holder and polished on cast iron for about $3 \mathrm{~h}$ using olive oil and diamond suspensions of 15,6 , and $1 \mu \mathrm{m}$ in size to have a smooth surface finish. All the diamonds were then polished on a regular polishing lap using diamond suspensions of 6 , followed by $1 \mu \mathrm{m}$ size. RMS roughness was measured to be less than $1.5 \mathrm{~nm}$ by FIB cross-section in the areas of interest for the measurement. The substrates were then rinsed clean with acetone, ethanol, and isopropanol. Though at this point the diamond surfaces are already H-terminated, ${ }^{19,39}$ the surface condition of the diamonds was modified using 2 additional and different treatments.

\section{Hydrogen plasma treatment}

This treatment was conducted in a Balzers BAI730D chamber, using a 95:5 Ar: $\mathrm{H}$ mixture at a pressure of $10^{-3}$ mbar. The plasma was produced between an ionization chamber using a hot tungsten filament at the top of the reactor and a copper cone at its bottom, using a DC current of $80 \mathrm{~A}$. The plasma had a columnar shape producing a radial diffusive flux of ions and radicals used for the treatment. The recombination enthalpy of the atomic $\mathrm{H}$ was used to heat up the diamond substrates. Their temperature was monitored by inserting thermocouples protected by a zirconia shield at the same distance from the plasma center. The obtained temperatures are somewhat approximate as the zirconia surface reactivity is not the same as that of diamond, but we expect the measured temperatures to be within $50{ }^{\circ} \mathrm{C}$ of the actual value for our diamonds. The diamonds were mounted on Ta sample holders coated with boron nitride to prevent the formation of tantalum carbide. The diamonds were inserted at $16 \mathrm{~cm}$ from the plasma center, reaching temperatures of $700^{\circ} \mathrm{C}$. The treatment conditions were maintained for $2 \mathrm{~h}$ to make sure the whole surface had reacted.

\section{RF etching treatment}

Samples were put in a Balzers BAS 450 sputter deposition system and a RF etching was conducted so as to remove the first few layers of carbon atoms, including the $\mathrm{H}$ termination, leading to surface termination with $\mathrm{sp}^{2}$ chains. ${ }^{19}$ Since the surface energy difference between diamond $2 \times 1$ and $1 \times 1$ terminated surfaces and $\mathrm{Al}$ is of the order of $1 \mathrm{eV}$ per unit cell ${ }^{30}$ in favor of the latter, we are confident that the deposition of a metallic layer afterwards changes the surface configuration.

\section{Layer deposition}

The samples were coated with $\mathrm{Ni}, \mathrm{Cu}$, and $\mathrm{Ti}$ layers of thicknesses of 63,57 , and $62 \mathrm{~nm}$, respectively, then with $\mathrm{Al}$ layers of 37, 40, and $45 \mathrm{~nm}$. Samples with a single Al layer of $105 \mathrm{~nm}$ ( $\mathrm{H}$ terminated diamond) and $145 \mathrm{~nm}\left(\mathrm{sp}^{2}\right.$ terminated diamond) were also prepared. $\mathrm{The} \mathrm{Ni}, \mathrm{Ti}$, and $\mathrm{Al}$ layers were all deposited in an Alcatel EVA 600 evaporator using e-beam evaporation at deposition speeds of 14, 12, and $11 \mathrm{~A} \mathrm{~s}^{-1}$. The $\mathrm{Cu}$ layer was deposited in the same evaporator using thermal evaporation in a tungsten crucible at a speed of $5 \AA \mathrm{s}^{-1}$. The layer thicknesses were measured with a $2 \mathrm{~nm}$ accuracy and their density verified using cross-section imaging in a Zeiss NVision 40 Focused Ion Beam. The top Al layers were found to be necessary as suscepting layers (except for the Ni/C:H sample, where a layer of $120 \mathrm{~nm} \mathrm{Ni}$ 
TABLE I. Comparison of the lattice constants $a$ in $\AA$ and bulk moduli $B$ in GPa of diamond, nickel, and copper. Literature values are from experiment [Exp].

\begin{tabular}{lccccc}
\hline \hline & \multicolumn{2}{c}{$a[\AA]$} & & \multicolumn{2}{c}{$B$ [GPa] } \\
\cline { 2 - 3 } \cline { 5 - 6 } Material & Present work & Literature [Exp] & Present work & Literature [Exp] \\
\hline Diamond & 3.574 & 3.560 (Ref. 45) & & 428 & 443 (Ref. 46) \\
Nickel & 3.518 & 3.523 (Ref. 47) & & 190 & $186-195$ (Ref. 48) \\
Copper & 3.638 & 3.624 (Ref. 49) & & 133 & 133 (Ref. 50) \\
\hline \hline
\end{tabular}

was deposited and the $\mathrm{Y}$ signal from the lock-in amplifier was large enough for the measurement to be valid) for the experiment because the thermoreflectance coefficients of $\mathrm{Cu}$, $\mathrm{Ni}$, and Ti investigated are $\mathrm{low}^{40}$ at the wavelength used in our TDTR setup and some of the measured out-of-phase signals were extremely low as compared to the in-phase signals, making measurement prone to artifacts without the $\mathrm{Al}$ layer.

\section{B. Theory}

We use DFT as implemented in the Vienna $A b$ initio Simulation Package ${ }^{41}$ to calculate the total energies of different interface structures. We approximate the exchangecorrelation energy by the PBE generalized gradient approximation $^{42}$ and use projector-augmented plane wave (PAW) potentials. ${ }^{43}$ A $11 \times 11 \times 11$ Monkhorst-Pack ${ }^{44}$ k-point sampling was used for the cubic unit cells of the metals and rescaled appropriately for the larger interface structures. An energy cutoff of $390 \mathrm{eV}$ was required to appropriately converge the system and the Methfessel-Paxton smearing of second order with a smearing width of $0.1 \mathrm{eV}$ was used. The forces of all structures were relaxed to a magnitude less than $10^{-3} \mathrm{eV} / \AA ̊ \AA$ using the conjugate gradient method.

Values for the lattice constant $a$ and bulk modulus $B$ were calculated by fitting the Birch-Murnaghan equation of state as implemented in the FHIMD Toolkit. ${ }^{51}$ The obtained values are compared against existing data in Table I. We compare the calculated surface energies for each material with literature values in Table II. We find that the lattice mismatch between the metal and diamond systems is very low, only $+1.8 \%$ and $-1.1 \%$ for $\mathrm{Cu}$ and $\mathrm{Ni}$, respectively. We therefore choose the lattice parameter of the diamond substrate as the lattice parameter for the interface system. This is a reasonable choice because diamond is much stiffer than the two metals (see Table I).

As a model system, we studied the (111) interface of $\mathrm{Cu}$ or Ni with diamond as shown in Fig. 1. Supercells were constructed to model the high-symmetry interface configuration in the [111] direction. There are three such configurations, labeled $A, B$, and $C$ in Fig. 1: in configuration $A$, the surface $\mathrm{C}$ atoms (or the $\mathrm{H}$ atoms when the diamond surface is hydrogen-terminated) are placed directly above the surface metal atoms; in configuration $B$, the metal atoms are directly below a second-layer $\mathrm{C}$ atom; in configuration $C$, the metal atoms are directly below the center of the puckered hexagons formed by first and second layer $\mathrm{C}$ atoms. The supercells were treated using periodic boundary conditions. The thickness of each diamond and metal layer was converged, so that the middle of each layer can be considered as bulk-like. In all cases, we considered a simple, unreconstructed unit cell with $1 \times 1$ periodicity relative to the bulk-terminated plane. This structure was chosen here even though the $2 \times 1$ Pandey chain reconstruction ${ }^{52}$ is energetically preferred on the diamond (111) clean surface. The reason for this choice is that the deposition of the metal on the clean diamond surface is expected to undo the chain reconstruction, resulting in an unreconstructed surface with the regular bulk-terminated periodicity. This expectation is borne out in experiment for the case of the $\mathrm{Al} /$ diamond interface, where it was shown that the work of adhesion is $61 \%$ higher in the case of the $1 \times 1$ configuration. ${ }^{30}$ We expect this difference to remain robust when using $\mathrm{Cu}$ or $\mathrm{Ni}$ as the metal layers deposited on the diamond surface, so that the simple $1 \times 1$ periodicity is the most stable configuration.

TABLE II. Work of adhesion $W_{\text {adh }}$ calculated by separating the metal-diamond interface (with and without $\mathrm{H}$ termination), for the four cases of interest, $\mathrm{Ni} / \mathrm{C}: \mathrm{H}, \mathrm{Ni} / \mathrm{C}, \mathrm{Cu} / \mathrm{C}: \mathrm{H}, \mathrm{Cu} / \mathrm{C}$. As a reference, we also include the surface energies of (111) planes for diamond (with and without hydrogen termination), Ni and $\mathrm{Cu}$, as calculated here and as reported in the literature; [DFT] indicates a theoretical value, [Exp] indicates and experimental value.

\begin{tabular}{|c|c|c|c|c|c|c|c|}
\hline \multirow[b]{2}{*}{ Surface } & \multicolumn{3}{|c|}{ Surface energy $\left[\mathrm{Jm}^{-2}\right]$} & \multicolumn{4}{|c|}{ Work of adhesion $\left[\mathrm{Jm}^{-2}\right]$} \\
\hline & Present work & Literature [DFT] & Literature [Exp] & Interface & A & B & $\mathrm{C}$ \\
\hline $\mathrm{C}(111)$ & 5.73 & 5.66 (Ref. 29) & & $\mathrm{Ni} / \mathrm{C}: \mathrm{H}$ & 0.10 & 0.09 & 0.11 \\
\hline $\mathrm{C}(111): \mathrm{H}$ & $<0.015$ & 0.04 (Ref. 29) & & $\mathrm{Ni} / \mathrm{C}$ & 5.00 & 3.95 & 3.75 \\
\hline $\mathrm{Ni}(111)$ & 2.01 & & 1.90 (Ref. 53) & $\mathrm{Cu} / \mathrm{C}: \mathrm{H}$ & 0.07 & 0.05 & 0.06 \\
\hline $\mathrm{Cu}(111)$ & 1.28 & 1.29 (Ref. 31) & 1.49 (Ref. 54) & $\mathrm{Cu} / \mathrm{C}$ & 3.04 & 3.01 & 3.00 \\
\hline
\end{tabular}
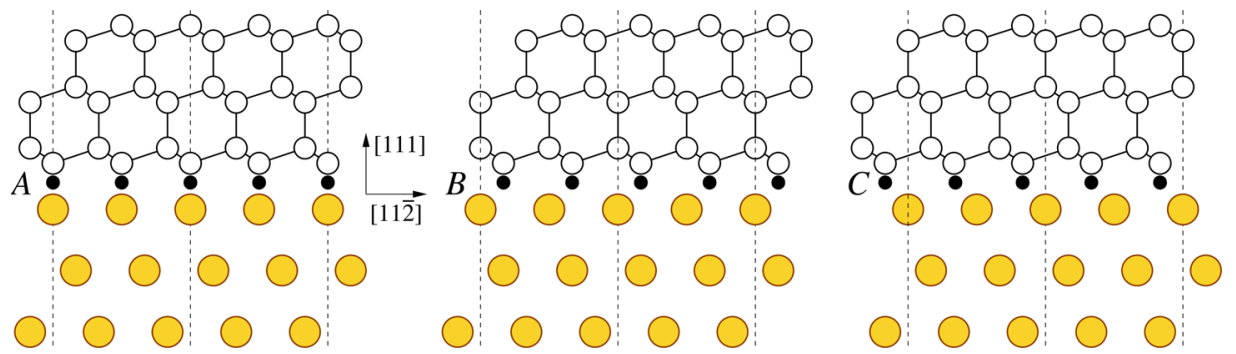

FIG. 1. Schematic representation of the three high-symmetry interface configurations considered here, which are labeled $A, B, C$ : carbon atoms are shown as open black circles, hydrogen atoms as smaller, filled black circles, and metal atoms as larger, yellow circles. The work of adhesion $W_{\text {adh }}$ is calculated by separating the diamond structure from the metal at the interface between the two. 

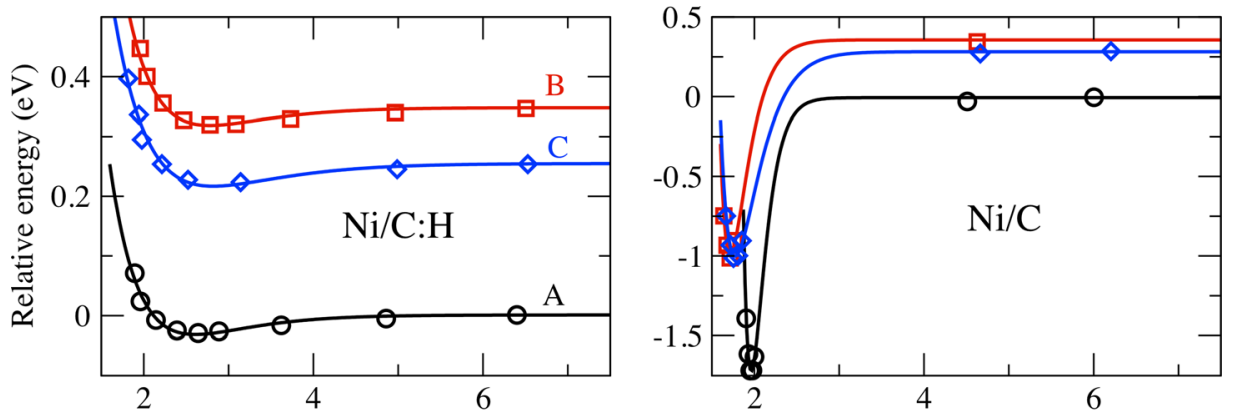

FIG. 2. Relative energy as a function of distance and configuration for the $\mathrm{Ni} / \mathrm{H}: \mathrm{C}, \mathrm{Ni} / \mathrm{C}, \mathrm{Cu} / \mathrm{H}: \mathrm{C}$, and $\mathrm{Cu} / \mathrm{C}$ interfaces. In each case, black circles, red squares, and blue diamonds correspond to the calculated energy of the configurations labeled $A, B, C$, respectively, in Fig. 1; the lines going through the calculated values are fits of the Universal Binding Energy Relation expression proposed by Rose et al. ${ }^{55-57}$ Although the universal binding energy relation has been initially developed specifically for metallic systems, ${ }^{55-57}$ it has since been shown to be a much more general relation for the cohesion of many types of solids. ${ }^{58}$ Curves for each structure are shifted by the value of the lowest energy configuration, which is configuration $A$ for all cases, in the limit when the two surfaces are completely separated.

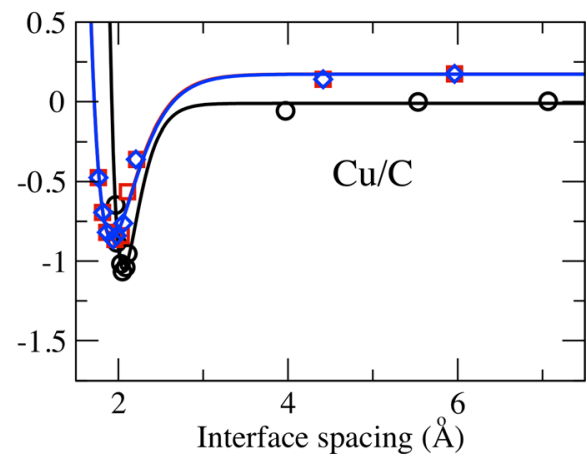

\section{RESULTS AND DISCUSSION}

Table II summarizes the results of the surface energies of the (111) planes of diamond (with or without $\mathrm{H}$ termination), $\mathrm{Ni}$ and $\mathrm{Cu}$, obtained by calculating one-half of the material's work of cohesion. The surface energies compare well with other literature results, with a maximal difference of less than $5 \%$ for the surface energy of Ni. We explain the discrepancy found between the DFT data on $\mathrm{Cu}$ and the experimental data from Ref. 54 by the fact that measurements were conducted at $927^{\circ} \mathrm{C}$, while DFT calculations yield the theoretical value at $0 \mathrm{~K}$.

Figure 2 shows the total energy as a function of distance between the diamond and metal surfaces. The fitting curves presented are obtained using the Universal Binding Energy Relation curve proposed by Rose et al. ${ }^{55-57}$

$$
E_{\mathrm{tot}}=E_{\mathrm{inf}}-E_{\mathrm{adh}}\left(1+\frac{x-x_{0}}{\lambda}\right) \exp \left[-\frac{x-x_{0}}{\lambda}\right]
$$

with $E_{\text {tot }}$ is the total energy of the system, $E_{\text {inf }}$ is the energy when the two surface are completely separated, $x$ is the distance between the surfaces, $x_{0}$ its equilibrium value, and $\lambda$ is a Thomas-Fermi screening length specific for the materials on each side of the interface. The adhesion energy is defined

TABLE III. Values of TBC measured by TDTR for the materials interfaces of interest.

\begin{tabular}{lc}
\hline \hline Materials couple & $\mathrm{TBC}\left[\mathrm{Wm}^{-2} \mathrm{~K}^{-1}\right]$ \\
\hline $\mathrm{Cu} / \mathrm{C}: \mathrm{H}$ & $29 \pm 3$ \\
$\mathrm{Al} / \mathrm{C}: \mathrm{H}$ & $32 \pm 3$ \\
$\mathrm{Ni} / \mathrm{C}: \mathrm{H}$ & $110 \pm 10$ \\
$\mathrm{Cu} / \mathrm{C}$ & $110 \pm 15$ \\
$\mathrm{Al} / \mathrm{C}$ & $180 \pm 20$ \\
$\mathrm{Ni} / \mathrm{C}$ & $310 \pm 35$ \\
$\mathrm{Ti} / \mathrm{C}$ & $330 \pm 20$ \\
\hline \hline
\end{tabular}

as the value of the minimum of the energy relative to the energy at infinite separation of the surfaces. To obtain the work of adhesion, the adhesion energy is converted in $\mathrm{Jm}^{-2}$ units and divided by twice the surface of the interface in $\mathrm{m}^{2}$. In order to have a direct comparison of the relative energy of the different configurations for the interface, labeled $A, B$, and $C$ in Fig. 1, we shifted all curves by the same amount. The constant energy shift was chosen so that the lowest energy configuration is set to zero in the limit of infinite separation. In all cases, the lowest energy configuration is the one labeled $A$ in Fig. 1. Table III gives the values for TBC for the interfaces investigated. The average value over 4 measurements is given and the error is taken to be the standard deviation over these measurements. Fig. 3 shows the ratio of the experimentally measured $\mathrm{TBCs}$ and the corresponding radiation limit $\left(\mathrm{TBC}_{\mathrm{RL}}\right)$ as a function of the work of adhesion calculated in this study (for $\mathrm{Ni} / \mathrm{C}: \mathrm{H}, \mathrm{Ni} / \mathrm{C}$, $\mathrm{Cu} / \mathrm{C}: \mathrm{H}$, and $\mathrm{Cu} / \mathrm{C}$ interfaces) and deduced from literature values (for $\mathrm{Al} / \mathrm{C}, \mathrm{Al} / \mathrm{C}: \mathrm{H}$, and $\mathrm{Ti} / \mathrm{C}$ (Refs. 29 and 31). The

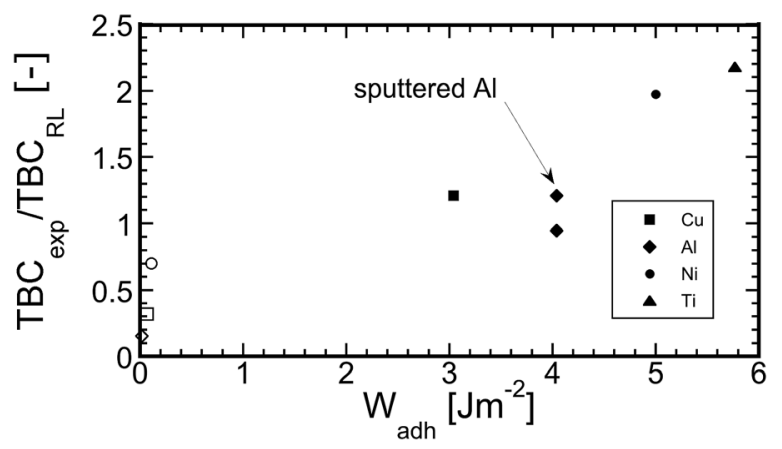

FIG. 3. Ratio of the measured $\mathrm{TBC}$ and radiation limit $\left(\mathrm{TBC}_{\mathrm{RL}}\right)$ of the materials on each side of the interface, as a function of the system's work of adhesion. Metals on hydrogenated diamond systems are shown in open symbols, while their corresponding full shapes are the metals on $\mathrm{sp}^{2}$-terminated diamonds. Data for $W_{\text {adh }}$ between Ti and diamond are from Guo et al. ${ }^{31}$ The data for an $\mathrm{Al}$ layer deposited by sputtering ${ }^{32}$ is shown. 
Ti/C:H case was not measured as there is no calculation of $W_{\text {adh }}$ for this interface.

Based on the observation that the computed values for the bulk moduli, lattice constants, and surface energies are in good agreement with experiment, we consider that our results for $W_{\text {adh }}$ are of sufficient accuracy to describe qualitatively the trends in the systems studied here. The only exception is the calculated value of the $\mathrm{C}(111): \mathrm{H}$ surface, but this surface is known to have an extremely low surface energy, which matches quantitatively that of other hydrocarbons, and thus the calculated $E_{\text {adh }}$ is of the order of $0.001 \mathrm{eV}$, which is below the accuracy of the procedure used (hence the " $<$ " in Table II).

The $W_{\text {adh }}$ values found for hydrogenated interfaces in Fig. 2 exhibit the trend already mentioned earlier, that is, the work of adhesion of both $\mathrm{Ni}$ and $\mathrm{Cu}$ on a hydrogenated diamond (111) surface is of the order of $0.1 \mathrm{Jm}^{-2}$. In all cases, the configuration $A$ is preferred energetically, so it should be the most stable structure. Interestingly, this configuration also has the highest work of adhesion, except in the case of the $\mathrm{Ni} / \mathrm{C}: \mathrm{H}$ system, in which configuration $C$ has higher work of adhesion, although the difference with $A$ is very small and probably not significant. In fact, hydrogen-terminated surfaces seem to always yield very low $\mathrm{W}_{\text {adh }}$, so the exact quantitative result should be taken cautiously; the crucial point here is that there is a factor of 30 to 50 differences in the values of $\mathrm{W}_{a d h}$ between interfaces with and without hydrogen. Figure 4 shows the electron densities close to the metal/diamond interface with and without interfacial hydrogen. Two observations arise: (i) when interfacial hydrogen is present, a negligible amount of electrons are shared between the metal and the carbon atoms, which explains the weak interfacial bonding as observed in

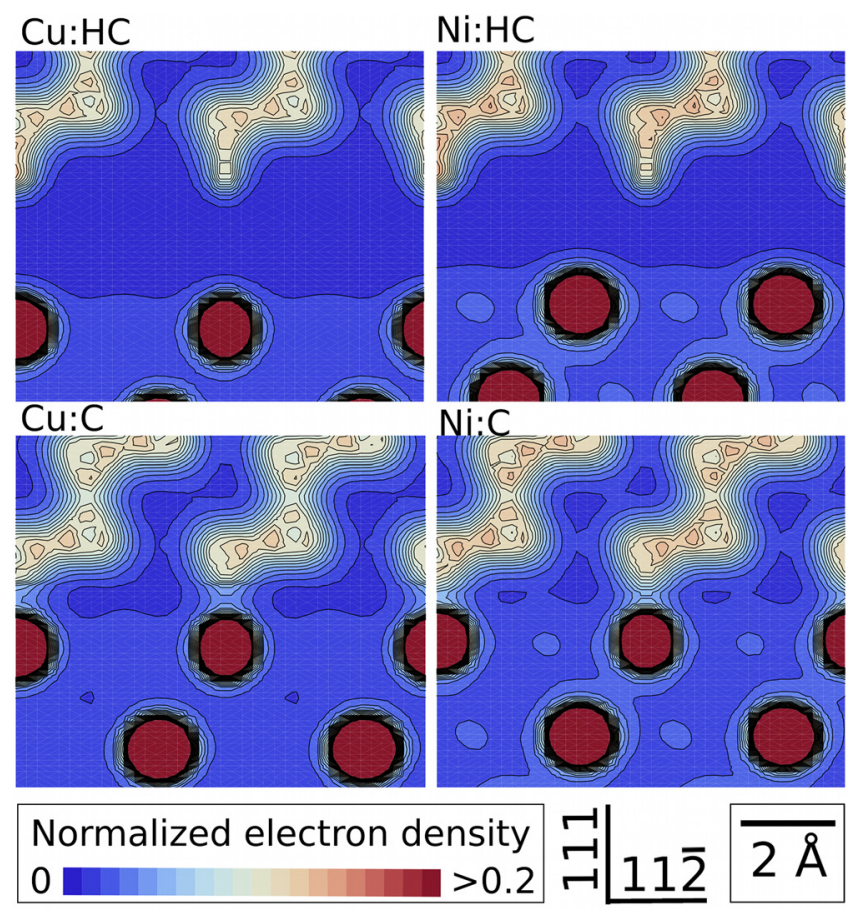

FIG. 4. Electron densities at the metal/diamond interface relative to maximum values for all the cases investigated, taken along the $(\overline{1} 10)$ plane.
Table II, (ii) more electrons are shared through the $\mathrm{Ni} / \mathrm{C}$ than through the $\mathrm{Cu} / \mathrm{C}$ interface, corroborating the lower work of adhesion found for the latter case (Table II). For the Ni/C case, we find a high $W_{\text {adh }}$ value of $5 \mathrm{Jm}^{-2}$, which is close to that of the Ti/C interface found by Guo et al. ${ }^{31} 5.77 \mathrm{Jm}^{-2}$. The fact that configuration $A$ has higher $W_{\text {adh }}$ in this case disagrees with the results by Pickett et al. ${ }^{59}$ who found that configuration $B$ has a higher $W_{\text {adh }}$ value. However, in that work, the interfacial distance was not optimized; as can be seen from Fig. 2, at the interplanar distance where configurations $B$ and $C$ have a minimum energy (approximately $1.75 \AA$ ), configuration $A$ is not at its equilibrium position, which is found at a separation of $1.96 \AA$.

Values presented in Table III show that hydrogen has a negative effect on the TBC and $\mathrm{W}_{a d h}$ between all the metals and diamond. The effect of hydrogen can be agrees with theoretical calculations by Prasher, ${ }^{60}$ showing that a reduction in interfacial bond strength by a factor of ten reduces TBC by the same factor at high temperatures. A notable point is that the $\mathrm{Ni} / \mathrm{C}: \mathrm{H}$ interface still exhibits a very high TBC compared what one obtains with $\mathrm{Al}$ and $\mathrm{Cu}$. This can be rationalized by the fact that Ni has a strong affinity for hydrogen compared to $\mathrm{Al}$ and $\mathrm{Cu}$. Comparing the hydrogen solubilities in these materials, we find $1.15 \times 10^{-9}$ (Ref. 61), $3.6 \times 10^{-7}$ (Ref. 62) and 1.4 (Ref. 63) atomic ppm for $\mathrm{Al}, \mathrm{Cu}$, and $\mathrm{Ni}$, respectively, at ambient conditions. This difference of 7 orders of magnitude means that $\mathrm{Ni}$ may dissolve non-negligible amounts of hydrogen at ambient conditions, while the two other metals do not. A metallic thin film can be highly constrained in tension and contain many grain boundaries, ${ }^{64}$ a fact that has been shown to further increase the low-temperature solubility by as much as an order of magnitude in $\mathrm{Ni}^{69}$

Figure 3 shows the ratio of measured TBCs and the corresponding Radiation Limit $\mathrm{RL}\left(\mathrm{h}_{b d, \mathrm{RL}}\right)$ calculated as follows, using the formulation proposed by Swartz and Pohl ${ }^{65}$ in the Debye approximation:

$$
h_{b d}=\frac{1}{8 \pi^{2}} \sum_{p} \int_{0}^{\omega_{m}} \hbar \omega^{3} c_{p, D}^{-2} \frac{\partial n_{\omega, p, T}}{\partial T} d \omega
$$

with $\omega_{m}$ the maximum phonon pulsation in the metal, $p$ the polarization, $T$ the temperature, and $c$ the sound velocity in diamond. In the high temperature limit, this expression can be written in the form proposed by Lyeo and Cahill ${ }^{16}$

$$
h_{b d, \mathrm{R} L}=\frac{1}{8 \pi^{2}} \sum_{p} k_{B} \frac{\omega_{m}^{3}}{3 c_{p, D}^{2}}=\frac{\pi k_{B} \nu_{m}^{3}}{c_{D}^{2}}
$$

with $k_{B}$ the Boltzmann constant, $\nu_{m}$ the maximum phonon frequency of the metal, and $c_{D}$ the average phonon group velocity in diamond. The Radiation Limit gives the maximum value of TBC if only elastic phonon transfer is assumed. Using the RL as a normalization factor hence has the goal of removing the influence of the elastic properties (as visible in the metal's $\nu_{m}$ ) of the materials on either sides of the interface, thereby highlighting possible effects due to other factors. The obtained ratio and $W_{\text {adh }}$ seem to be qualitatively linked, with an almost linear relation. Indeed, three other 
factors can potentially impact TBC: (i) the change in atomic mass across the interface, (ii) the interface's roughness, and (iii) differences in the electron-phonon coupling constant between the metals. Argument (i) can be ruled out as $\mathrm{Cu}$ and Ni have similar atomic masses (58.69 and $63.55 \mathrm{amu}$, respectively, to be compared with 12.01 for $\mathrm{C}$ atoms) and have widely different $\mathrm{TBCs}$ with diamond; conversely, $\mathrm{Ni}$ and $\mathrm{Ti}$ have moderately different atomic masses (58.69 and $47.87 \mathrm{amu}$, respectively) and very close TBC values. The effect of interface roughness (argument ii) should not be significant as the roughness of the zones investigated was measured to be below $2 \mathrm{~nm}$, a value that has been shown to have only a weak influence, at least on an $\mathrm{Al} / \mathrm{Si}$ interface. ${ }^{66}$ Moreover, the same polishing procedure was used for all the diamonds and therefore a roughness varying wildly from sample to sample cannot be expected. Argument (iii) assumes that in order for a thermal flux to flow through a metal-dielectric interface, electrons have first to thermalize with phonons close to the interface, and that the so-produced phonons can then be transmitted through it. This effect, described by Majumdar and Reddy, ${ }^{67}$ can be rationalized by a difference between electrons and lattice temperature close to the interface that depends on the phonon thermal conductivity $k_{p}$ of the metal and its electron-phonon coupling constant $g$. However, using the formula for the electron-phonon part of the interface conductance provided in Ref. 67, $h_{e-p}=\sqrt{g k_{p}}$, taking conservative values of $k_{p}$ around $20 \mathrm{Wm}^{-1} \mathrm{~K}^{-1}$ and $g$ of $10^{17} \mathrm{Wm}^{-3} \mathrm{~K}^{-1}$ (the value for copper, from Ref. 68), the calculated value falls above $1 \mathrm{GWm}^{-2} \mathrm{~K}^{-1}$, well above the measured value and hence not affecting them in a critical way.

The only exception to the observed correlation between $\mathrm{W}_{\text {adh }}$ and $\mathrm{TBC}$ seems to be $\mathrm{Al} / \mathrm{C}$, but this system shows large differences in TBC values depending on the deposition technique used, ${ }^{32}$ exemplified by the value of $230 \pm 35$ $\mathrm{MWm}^{-2} \mathrm{~K}^{-1}$ obtained for the same system with a sputtered $\mathrm{Al}$ layer, ${ }^{32}$ which in itself reinforces this work's argument as sputtered layers are expected to adhere better to a substrate than evaporated ones. ${ }^{64}$

We attribute the very high conductances measured to the presence of interfacial states due to the strong bonding which has been suggested to impact the transmissivity of an interface. $^{70,73}$ This is due to the existence of modes that are intermediate between those of the substrate and metallic layer, which is thought to be beneficial to $\mathrm{TBC} .^{71,72,74}$ Calculations of phonon spectra close to or at the interface ${ }^{75}$ could be useful in further elucidating this issue.

\section{CONCLUSION}

We performed both experimental studies and theoretical investigations, based on DFT, to determine the work of adhesion $W_{\text {adh }}$ of high symmetry configurations of the $\mathrm{Cu}$ and $\mathrm{Ni}$ interfaces with diamond in the [111] direction, with and without interfacial hydrogen. We find that hydrogen decreases very significantly the work of adhesion between the metal and diamond, in the case of $\mathrm{Cu}$ from 3.04 to 0.07 $\mathrm{Jm}^{-2}$ and in the case of $\mathrm{Ni}$ from 5.00 to $0.11 \mathrm{Jm}^{-2}$. Hydrogen has a similar impact on the $\mathrm{M} /$ diamond Thermal
Boundary Conductance $(\mathrm{M}=\mathrm{Al}, \mathrm{Cu}, \mathrm{Ni})$. This trend is captured by a qualitative link between the work of adhesion and the TBC of an interfacial system: the higher the work of adhesion, the higher the ratio between the experimentally measured TBC and the corresponding theoretical Radiation Limit. We suggest that the reason for the increase in TBC obtained for high values of the work of adhesion is related to the formation of interfacial phononic states due to strong bonding.

\section{ACKNOWLEDGMENTS}

C. Monachon gratefully acknowledges financial support by the SNSF Project Nos. 200021-121881 and 200020135132. The authors kindly acknowledge Christof Hollenstein and Loï Curchod for their time and advice as well as for the Ar:H plasma treatments. We acknowledge Professor Hubert Girault of the Laboratoire d'Électrochimie Physique et Analytique (LEPA) at EPFL for providing the laser source for the experiments.

${ }^{1}$ O. Beffort, F. A. Khalid, L. Weber, P. Ruch, U. E. Klotz, S. Meier, and S. Kleiner, Diam. Relat. Mater. 15, 1250-1260 (2006).

${ }^{2}$ L. Weber and R. Tavangar, Scr. Mater. 57, 988-991 (2007).

${ }^{3}$ W. S. Capinski, H. J. Maris, T. Ruf, M. Cardona, K. Ploog, and D. S. Katzer, Phys. Rev. B 59, 8105 (1999).

${ }^{4}$ P. E. Hopkins, J. C. Duda, S. P. Clark, C. P. Hains, T. J. Rotter, L. M. Phinney, and G. Balakrishnan, Appl. Phys. Lett. 98, 161913 (2011).

${ }^{5}$ K. E. Goodson and Y. S. Ju, Annu. Rev. Mater. Sci. 29, 261 (1999).

${ }^{6}$ D. G. Cahill, K. E. Goodson, and A. Majumdar, J. Heat Transf. 124, 223 (2002).

${ }^{7}$ D. G. Cahill, F. K. Wayne, K. E. Goodson, G. D. Mahan, A. Majumdar, H. J. Maris, R. Merlin, and S. R. Philipot, Appl. Phys. Rev. 93, 793 (2003).

${ }^{8}$ E. Pop and K. E. Goodson, J. Electron. Packag. 128, 102 (2006).

${ }^{9}$ R. M. Costescu, D. G. Cahill, F. H. Fabreguette, Z. A. Sechrist, and S. M. George, Science 303, 989 (2004).

${ }^{10}$ C. Chiritescu, D. G. Cahill, N. Nguyen, D. Johnson, A. Bodapati, P. Keblinski, and P. Zschack, Science 315, 351 (2007).

${ }^{11}$ P. E. Hopkins, P. M. Norris, R. J. Stevens, T. E. Beechem, and S. Graham, J. Heat Transf. 130, 062402 (2008).

${ }^{12}$ B. C. Gundrum, D. G. Cahill, and R. S. Averback, Phys. Rev. B 72, 245426 (2005).

${ }^{13}$ P. E. Hopkins, T. E. Beechem, J. C. Duda, K. Hattar, J. F. Ihlefeld, M. A. Rodriguez, and E. S. Piekos, Phys. Rev. B 84, 125408 (2011).

${ }^{14}$ P. E. Hopkins, J. C. Duda, C. W. Petz, and J. A. Floro, Phys. Rev. B 84, 035438 (2011).

${ }^{15}$ J. C. Duda and P. E. Hopkins, Appl. Phys. Lett. 100, 111602 (2012).

${ }^{16}$ H.-K. Lyeo and D. G. Cahill, Phys. Rev. B 73, 144301 (2006).

${ }^{17}$ K. C. Collins, S. Chen, and G. Chen, Appl. Phys. Lett. 97, 083102 (2010).

${ }^{18}$ C. Monachon, M. Hojeij, and L. Weber, Appl. Phys. Lett. 98, 091905 (2011).

${ }^{19}$ C. Monachon and L. Weber, J. Appl. Phys. 113, 183504 (2013).

${ }^{20}$ M. D. Losego, M. E. Grady, N. R. Sottos, D. G. Cahill, and P. V. Braun, Nature Mater. Lett. 11, 502 (2012).

${ }^{21}$ S. A. Varchenya, A. Simanovskis, and S. V. Stolyarova, Thin Solid Films 164, 147 (1988).

${ }^{22}$ A. A. Volinsky, N. R. Moody, and W. W. Gerberich, Acta Mater. 50, 441 (2002).

${ }^{23}$ B. Schwarz, C. Schrank, C. Eisenmenger-Sittner, M. Stöger-Pollach, M. Rosner, and E. Neubauer, Surf. Coat. Technol. 200, 4891 (2006).

${ }^{24}$ H. M. Jensen, Eng. Fract. Mech. 40, 475 (1991).

${ }^{25}$ J. W. Hutchinson, M. D. Thouless, and E. G. Liniger, Acta Metall. Mater. 40, 295 (1992).

${ }^{26}$ M. W. Moon, H. M. Jensen, J. W. Hutchinson, K. H. Oh, and A. G. Evans, J. Mech. Phys. Solids 50, 2355 (2002).

${ }^{27}$ S. J. Bull, Surf. Coat. Technol. 50, 25 (1991).

${ }^{28}$ A. G. Evans, J. W. Hutchinson, and Y. Wei, Acta Mater. 47, 4093 (1999). 
${ }^{29}$ Y. Qi and L. G. Hector, Phys. Rev. B 69, 235401 (2004).

${ }^{30}$ Y. Qi and L. G. Hector, Phys. Rev. B 68, 201403 (2003).

${ }^{31}$ H. Guo, Y. Qi, and X. Li, J. Appl. Phys. 107, 033722 (2010).

${ }^{32}$ C. Monachon and L. Weber, Diam. Relat. Mater. 39, 8 (2013).

${ }^{33}$ P. E. Hopkins, M. Baraket, E. V. Barnat, T. E. Beechem, S. P. Kearney, J. C. Duda, J. T. Robinson, and S. G. Walton, Nano Lett. 12, 590 (2012).

${ }^{34}$ K. Kang, Y. K. Koh, C. Chiritescu, X. Zheng, and D. G. Cahill, Rev. Sci. Instrum. 79, 114901 (2008).

${ }^{35}$ C. Monachon and L. Weber, Emerg. Mat. Res. 1, 89 (2012).

${ }^{36}$ R. M. Costescu, M. A. Wall, and D. G. Cahill, Phys. Rev. B. 67, 054302 (2003).

${ }^{37}$ D. G. Cahill, Rev. Sci. Instrum. 75, 5119 (2004).

${ }^{38}$ A. J. Minnich, J. A. Johnson, A. J. Schmidt, K. Esfarjani, M. S. Dresselhaus, K. A. Nelson, and G. Chen, Phys. Rev. Lett. 107, 095901 (2011).

${ }^{39}$ H. Kawarada, Surf. Sci. Rep. 26, 205 (1996).

${ }^{40}$ Y. Wang, J. Y. Park, Y. K. Koh, and D. G. Cahill, J. Appl. Phys. 108, 043507 (2010).

${ }^{41}$ G. Kresse and J. Furthmüller, Phys. Rev. B 54, 11169 (1996).

${ }^{42}$ J. P. Perdew, K. Burke, and M. Ernzerhof, Phys. Rev. Lett. 77, 3865 (1996).

${ }^{43}$ G. Kresse and D. Joubert, Phys. Rev. B 59, 1758 (1999).

${ }^{44}$ H. J. Monkhorst and J. D. Pack, Phys. Rev. B 13, 5188 (1976).

${ }^{45}$ D. P. Riley, Nature 153, 587 (1944).

${ }^{46}$ H. J. McSkimin and P. Andreatch, J. Appl. Phys. 43, 2944 (1972).

${ }^{47}$ A. Leineweber, H. Jacobs, and S. Hull, Inorg. Chem. 40, 5818 (2001).

${ }^{48}$ H. M. Ledbetter and R. P. Reed, J. Phys. Chem. Ref. Data 2, 531 (1973).

${ }^{49}$ H. M. Otte, J. Appl. Phys. 32, 1536 (1961).

${ }^{50}$ W. B. Daniels and C. S. Smith, Phys. Rev. 111, 713 (1958).

${ }^{51}$ M. Bockstedte, A. Kley, J. Neugebauer, and M. Scheffler, Comput. Phys. Commun. 107, 187 (1997).

${ }^{52}$ K. C. Pandey, Phys. Rev. B 25, 4338 (1982).

${ }^{53}$ P. S. Maiya and J. M. Blakely, J. Appl. Phys. 38, 698 (1967).
${ }^{54}$ C. E. Bauer, R. Speiser, and J. P. Hirth, Met. Trans. A 7, 75 (1976).

${ }^{55}$ J. H. Rose, J. Ferrante, and J. R. Smith, Phys. Rev. Lett. 47, 675 (1981).

${ }^{56}$ J. H. Rose, J. R. Smith, and J. Ferrante, Phys. Rev. B 28, 1835 (1983).

${ }^{57}$ J. H. Rose, J. R. Smith, F. Guinea, and J. Ferrante, Phys. Rev. B 29, 2963 (1984).

${ }^{58}$ E. Kaxiras, Atomic and Electronic Structure of Solids (Cambirdge University Press, 2003).

${ }^{59}$ W. E. Pickett, M. R. Pederson, K. A. Jackson, and S. C. Erwin, Mater. Sci. Eng. B 14, 87 (1992).

${ }^{60}$ R. Prasher, Appl. Phys. Lett. 94, 041905 (2009).

${ }^{61}$ M. Ichimura, I. Mamoru, and M. Hayakawa, J. Jpn I. Met. Mater. 43, 876 (1979).

${ }^{62}$ R. B. McLellan, J. Phys. Chem. Sol. 34, 1137 (1973).

${ }^{63}$ R. B. McLellan and W. A. Gates, Acta Metall. 21, 181 (1973).

${ }^{64}$ C. V. Thompson, Annu. Rev. Mater. Sci. 30, 159 (2000).

${ }^{65}$ E. T. Swartz and R. O. Pohl, Rev. Mod. Phys. 61, 605 (1989).

${ }^{66}$ P. E. Hopkins, L. M. Phinney, J. R. Serrano, and T. E. Beechem, Phys. Rev. B 82, 085307 (2010).

${ }^{67}$ A. Majumdar and P. Reddy, Appl. Phys. Lett. 84, 4768 (2004).

${ }^{68}$ J. Hohlfeld, S.-S. Wellershoff, J. Guedde, U. Conrad, V. Jaehnke, and E. Matthias, Chem. Phys. 251, 237 (2000).

${ }^{69}$ S. W. Stafford and R. B. McLellan, Acta Metall. 22, 1463 (1974).

${ }^{70}$ C. B. Saltonstall, C. A. Polanco, J. C. Duda, A. W. Ghosh, P. M. Norris, and P. E. Hopkins, J. Appl. Phys. 113, 013516 (2013).

${ }^{71}$ Z. Liang and H.-L. Tsai, J. Phys. Condens. Matter 23, 495303 (2011).

${ }^{72}$ T. S. English, J. C. Duda, J. L. Smoyer, D. A. Jordan, P. M. Norris, and L. V. Zhigilei, Phys. Rev. B 85, 035438 (2012).

${ }^{73}$ J. C. Duda, C.-Y. P. Yang, B. M. Foley, R. Cheaito, D. L. Medlin, R. E. Jones, and P. E. Hopkins, Appl. Phys. Lett. 102, 081901 (2013).

${ }^{74} \mathrm{C}$. Monachon and $\mathrm{L}$. Weber, "Influence of a nanometric $\mathrm{Al}_{2} \mathrm{O}_{3}$ interlayer on the thermal conductance of an $\mathrm{Al} /(\mathrm{Si}$,Diamond) interface," Adv. Eng. Mater. (submitted).

${ }^{75}$ Y. Chalopin and S. Volz, Appl. Phys. Lett. 103, 051602 (2013). 